\title{
Water-Wax Behaviour in Porous Silica at Low Temperature Fischer-Tropsch Conditions
}

Qingyuan Zheng, Jacob L. Brown, Mick D. Mantle, Andrew J. Sederman, T.A. Baart, C.M. Guédon and Lynn F. Gladden

\author{
University of Cambridge \\ Department of Chemical Engineering and Biotechnology \\ West Cambridge Site \\ Philippa Fawcett Drive \\ Cambridge CB3 OAS \\ United Kingdom
}

Submitted: 30 September 2018

Revised: 15 November 2018 


\begin{abstract}
Water is a major product of Fischer-Tropsch synthesis, and hence the behaviour of water within Fischer-Tropsch synthesis catalysts and its potential influence on catalyst rate and selectivity are questions of long-standing interest. The present work applies three different magnetic resonance techniques to study how water interacts with a model wax, $n$-octacosane, within the pore space of a porous silica of mean pore size $\sim 18 \mathrm{~nm}$. ${ }^{1} \mathrm{H}$ magnetic resonance spectroscopy, spin-lattice relaxation time and pulsed-field gradient measurements were performed at $195{ }^{\circ} \mathrm{C}$, and for water pressure in the range 3-13.6 bar, conditions relevant to low temperature FischerTropsch synthesis. The uptake of water within this system is shown to be very similar to that observed for capillary condensation of water within the empty pore space of the same porous silica under the same experimental conditions; suggesting that capillary condensation of water within the wax-saturated pores is occurring. The behaviour of water is characterised by two regimes. At low water relative pressures of $\sim 0.3 \leq P / P_{0} \leq \sim 0.8$ water moves into the pore space, displacing wax from the pore surface and existing as a water-rich layer between the pore surface and an oil-rich phase in the centre of the pore; the strong interaction with the pore surface is evidenced by the short nuclear spin relaxation time values of water at the lowest pressures which then increase slightly as multi-layer adsorption at the pore surface occurs with increase in pressure. In the water relative pressure range $\sim 0.8 \leq P / P_{0} \leq \sim 0.97$, condensation of water within the pores is observed, characterised by increases in both spin-lattice relaxation time and molecular diffusivity. Analysis of the data suggests that as much as $\sim 40 \%$ of the pore surface is occupied by condensed water after condensation has occurred. It is suggested that these two regimes of water behaviour inside initially wax-filled pores might explain previously reported aspects of the behaviour of Fischer-Tropsch catalyst performance as a function of pore size and amount of co-fed water.
\end{abstract}

Keywords: Fischer-Tropsch synthesis; water; phase behaviour; porous media; NMR 


\section{Introduction}

Fischer-Tropsch (FT) synthesis is a heterogeneous catalytic process that produces primarily linear hydrocarbons from synthesis gas (mixtures of $\mathrm{CO}$ and $\mathrm{H}_{2}$ ), and is central to the industrial gas-to-liquid (GTL) process [1], as well as of increasing interest in biomass-to-liquid (BTL) conversions [2]. Assuming conversion through to linear alkanes, the FT conversion can be expressed as:

$$
\mathrm{nCO}+(2 \mathrm{n}+1) \mathrm{H}_{2} \rightarrow \mathrm{C}_{\mathrm{n}} \mathrm{H}_{2 \mathrm{n}+2}+\mathrm{nH}_{2} \mathrm{O}
$$

FT reactions can be classified by operating conditions as low temperature FT (LTFT, 200$240{ }^{\circ} \mathrm{C}$ ) catalysed by $\mathrm{Co}$ and $\mathrm{Ru}$ catalysts and high temperature FT (HTFT, 300-350 ${ }^{\circ} \mathrm{C}$ ) catalysed by Fe-based catalysts [3]. LTFT, the conditions of which are considered in the present work, has a high conversion to heavy paraffin and has been commercially operated by Shell and Sasol in fixed-bed or slurry-bed reactors [1].

As is seen from Eq. 1, water is the primary by-product of FT synthesis, with one water molecule being produced for every carbon atom in the hydrocarbon chain. It is therefore expected that water might have a significant influence on the performance of FT catalysts. Indeed, water is known to affect the performance of both $\mathrm{Co}$ and Ru catalysts. In the case of Co catalysts, it has been reported that water can deactivate Co catalysts by causing surface oxidation [4-6], inducing Co sintering [7,8], and kinetically inhibiting adsorption of syngas [9]. It has also been proposed that water can enhance $\mathrm{CO}$ conversion on Co catalysts by kinetically increasing the amount of active surface carbon required for chain growth [10,11], and by enhancing intraparticle transport of syngas [12]. Water is also reported to have a positive influence on Co catalyst selectivity in that it increases the selectivity to $\mathrm{C}_{5+}$ products and reduces $\mathrm{CH}_{4}$ selectivity $[13,14]$. Considering $\mathrm{Ru}$ catalysts, water has generally been reported to kinetically enhance catalyst activity and selectivity by similar mechanisms to those proposed for Co $[11,15]$. The effect of water is observed to be a function of the amount of water present in the reaction system, either when produced during the reaction itself or when added in feed mixtures. Hibbitts et al. [11] observed an increase in the activity of a $\mathrm{Ru} / \mathrm{SiO}_{2}$ catalyst in a fixed-bed reactor with increasing residence time of the reactant which increased the amount of water generated by the reaction. Li et al. [9] studied the effect of water on a Pt promoted $\mathrm{Co} / \mathrm{Al}_{2} \mathrm{O}_{3}$ catalyst in a CSTR. They observed reversible deactivation when $<25 \mathrm{vol} \%$ of water was added 
to the feed, whilst for water content in the feed mixture $>25$ vol\% a sudden drop in $\mathrm{CO}$ conversion and irreversible deactivation was observed. The sudden change to significant catalyst deactivation at high water content in the reaction system was also observed on a $\mathrm{Co} / \mathrm{SiO}_{2}$ catalyst in a CSTR [14] and on $\mathrm{Co} / \mathrm{Al}_{2} \mathrm{O}_{3}$ catalysts in fixed-bed reactors $[16,17]$. The effect of water has also been found to be influenced by the pore structure of the support material used, with catalysts characterised by small pore size (pore size $\leq 10 \mathrm{~nm}$ ) tending to be associated with greater deactivation $[16,18,19]$.

The effect of water on FT catalyst performance is usually correlated to the partial pressure of water in a FT reactor [20]. However, it is important to recognise that it is the water inside the catalyst pellet (i.e., intra-pellet water), which is in equilibrium with water in the void space between the catalyst pellets (i.e., inter-pellet space), that contacts and influences the catalyst surface directly. Therefore, knowledge of the phase behaviour and properties of water within the catalyst pores is essential for understanding and quantifying effects of water on FT catalysts. It is well known that the phase behaviour and properties of fluids confined in pores are significantly different from those of bulk fluids due to the confinement effect of the pores and fluid-surface interactions at the pore surface [21,22]. However, the phase behaviour of water within the pore space of the FT catalyst under reaction conditions, and how water coexists with an immiscible wax phase existing within the pores under these conditions are not known. Despite the importance of the phase behaviour of water inside FT catalysts, there exist few publications on this topic, and these have addressed modelling and simulation studies. Ermolaev et al. $[23,24]$ considered the capillary condensation of water in FT catalyst pores in the numerical modelling of a FT catalyst and reactor. It was predicted that the capillary condensation of water could greatly influence catalyst performance under the reaction conditions by causing significant reduction in surface area available for reactants due to the low solubility of $\mathrm{CO}$ and $\mathrm{H}_{2}$ in water [23,24]. Papavasileiou et al. [25] studied the phase behaviour of a water and $n$-octacosane $\left(n-\mathrm{C}_{28}\right)$ mixture in $\mathrm{TiO}_{2}$ nanopores using molecular dynamics simulation. They reported that $n-\mathrm{C}_{28}$ was isolated from the surface by water which, as the surface-wetting phase, showed a density profile and diffusivity significantly different from those of bulk water as a function of distance from the pore wall [25].

This paper reports the first experimental study of water-wax phase behaviour within a porous material at conditions relevant to LTFT. The system studied is a fixed bed of porous $\mathrm{SiO}_{2}$ saturated by $n-\mathrm{C}_{28}$ at $195^{\circ} \mathrm{C}$ and at water vapour pressures of 3-13.6 bar. The pure oxide 
material is used so that the wax-water phase behaviour influenced by only the pore structure and silica surface chemistry is studied. In future work, modifications to the pore size and surface chemistry of the $\mathrm{SiO}_{2}$ studied here will be considered. $n-\mathrm{C}_{28}$ is chosen to model the FT wax as in previous studies [25,26]. The system is studied using three operando NMR techniques: ${ }^{1} \mathrm{H}$ NMR spectroscopy, ${ }^{1} \mathrm{H}$ pulsed-field gradient (PFG) NMR, and ${ }^{1} \mathrm{H}$ spin-lattice relaxation time $\left(T_{1}\right)$ measurements. ${ }^{1} \mathrm{H}$ NMR spectroscopy provides information of the amount of water and wax in pores at various water vapour pressures. PFG NMR has been widely applied to study the diffusion of molecules in porous media $[27,28]$ and in the present study it is used to measure diffusivities of water and wax during the water condensation process. Alongside these measurements, NMR $T_{1}$ measurements are used to characterise changes in the surface interaction of water and $n-\mathrm{C}_{28} ; T_{1}$ measurements are established as a useful indicator of molecule-surface interactions $[29,30]$.

\section{Experimental}

\subsection{Materials and experimental setup}

Porous silica spheres with diameters in the range 1.7-4 mm were used (Fuji Silysia Chemical); the silica spheres had a BET surface area and a BJH pore diameter of $171.6 \mathrm{~m}^{2} \mathrm{~g}^{-1}$ and $18.2 \mathrm{~nm}$ respectively, as measured by nitrogen adsorption analysis. The pore volume was $0.96 \mathrm{~cm}^{3} \mathrm{~g}^{-1}$. The nitrogen adsorption and desorption isotherms are shown in Fig. 1. Water uptake in the asreceived silica spheres (i.e., condensation of water into an empty pore space) and the same silica pre-saturated with $n$ - $\mathrm{C}_{28}$ (i.e., water condensing into a $n$ - $\mathrm{C}_{28}$-filled pore space) was studied. Hereafter, the experiment in which water uptake into the as-received silica spheres such that the pore space is empty when water is introduced is referred to as the water condensation reference experiment. The empty silica pellets used in the reference experiment were first dried in the oven overnight at $150{ }^{\circ} \mathrm{C}$ before the measurement. To prepare the $n-\mathrm{C}_{28}$ pre-saturated spheres for the water- $\mathrm{C}_{28}$ phase behaviour experiment, the as-received spheres were first dried in an oven at $150{ }^{\circ} \mathrm{C}$ for $12 \mathrm{~h}$ to remove surface physically-adsorbed water. $n$ $\mathrm{C}_{28}$ (Acros Organics, purity > 99\%) was then melted to liquid at $90{ }^{\circ} \mathrm{C}$ on a hot plate and the dried silica pellets were soaked in an excess amount of $n-\mathrm{C}_{28}$ liquid at the same temperature to saturate the pores for at least $12 \mathrm{~h} .0 .78 \mathrm{~g}$ of $n-\mathrm{C}_{28}$ was incorporated per gram of silica pellets, confirming that the pore volume $\left(0.96 \mathrm{~cm}^{3} \mathrm{~g}^{-1}\right)$ of the silica was fully saturated with $n-\mathrm{C}_{28}$ wax 
using this method. Before performing any NMR measurements on the $n-\mathrm{C}_{28}$ saturated pellets, the pellets were placed on filter paper pre-soaked with $n-\mathrm{C}_{28}$ liquid to remove the excess liquid on the external surface of the pellets without removing the liquid inside the pores. Deionised water was used in both experiments (ELGA PURELAB option-R7).

The water- $\mathrm{C}_{28}$ phase behaviour experiment was carried out in a pilot-scale flow loop designed to be operated at conditions up to $250{ }^{\circ} \mathrm{C}$ and 30 barg as shown in Fig. 2. The reactor and flow loop used have previously been described in application to the magnetic resonance imaging study of the heterogeneous catalytic oligomerization of ethene $[31,32]$.

Liquid water was delivered to the rig from a $2 \mathrm{~L}$ feed vessel by a HPLC piston pump. The volumetric flow rate of liquid was measured to an accuracy of $\pm 0.5 \mathrm{ml} \mathrm{h}^{-1}$ by a Coriolis meter. Nitrogen gas was delivered to the rig from a pressurised gas cylinder and the flow rate was controlled by a mass flow controller calibrated (with air) to measure a flow range of 3-60 NL $\mathrm{h}^{-1}$, with an accuracy of $\pm 0.01 \mathrm{NL} \mathrm{h}^{-1}$. A cylindrical pressure vessel within which the experiment was performed was constructed of silicon nitride $\left(\mathrm{Si}_{3} \mathrm{~N}_{4}\right)$, a non-magnetic ceramic material. The vessel was of inner diameter $20 \mathrm{~mm}$ and was placed in the vertical bore of the NMR magnet. To avoid contamination of the flow loop with displaced $n-\mathrm{C}_{28}$, the wax-saturated spheres were packed to a height of $20 \mathrm{~mm}$ within a glass tube of inner and outer diameter of $14.4 \mathrm{~mm}$ and $18 \mathrm{~mm}$, respectively. The glass tube was contained within the $\mathrm{Si}_{3} \mathrm{~N}_{4}$ pressure vessel, and was located such that the centre of the silica bed was located at the centre of the NMR radio frequency (r.f.) detection coil. The glass tube was designed with 4 holes of $5 \mathrm{~mm}$ in diameter in the wall of the tube to enable flow of nitrogen gas or water vapour from the $\mathrm{Si}_{3} \mathrm{~N}_{4}$ pressure vessel into the glass tube and hence into the bed of the porous silica spheres; pressure equilibrium inside and outside the glass tube being achieved. The silica bed was supported by a glass tripod of $30 \mathrm{~mm}$ in height with a $20 \mathrm{~mm}$ layer of glass wool located under the glass tripod. The glass tube was then sealed at the bottom by a detachable stainless steel cap which could be removed to allow re-packing the tube and for collecting the $n$ - $\mathrm{C}_{28}$ replaced by condensed water during the experiment. The effluent from the pressure vessel flowed to the waste collection vessel via the top and bottom return lines for upflow and downflow operations, respectively. The rig was operated in upflow mode for water flow and downflow mode for nitrogen flow. Switching between flow modes was controlled by three-way valves at the top and bottom of the pressure vessel. 
The lines connected to the top and the bottom of the pressure vessel, the sections of the vessel above and below the signal detection region, and the return lines were trace heated by electrical trace heaters to maintain system temperature. The trace heated regions are highlighted in Fig. 2. The temperature inside the pressure vessel was measured by two titanium-shielded thermocouples (TE) located above and below the signal detection region in the centre of the vessel. These thermocouples, along with the thermocouples fitted on the external surface of the pressure vessel (not shown in Fig. 2), controlled the temperature in the vessel to an accuracy of $\pm 1{ }^{\circ} \mathrm{C}$. Pressure control, to an accuracy of \pm 0.2 bar, was achieved by two parallel Badger RC-200 pressure control valves installed on the return line and the pressure transducer (PT) fitted on the top of the pressure vessel which measured the pressure inside the column. The control of the whole rig was performed using a LABVIEW application.

\subsection{Phase behaviour experiments}

All experiments and NMR measurements were performed at $195 \pm 1{ }^{\circ} \mathrm{C}$. This temperature was selected for the following reasons. First, the temperature was chosen to be as close to the reaction temperature of LTFT $\left(200-240^{\circ} \mathrm{C}\right)$ as possible while still allowing good temperature and pressure control of the system. Second, the saturation vapour pressure of $n-C_{28}$ is very low $\left(3.4 \times 10^{-4}\right.$ bar at $\left.195^{\circ} \mathrm{C}[33]\right)$ such that the evaporation of $n-\mathrm{C}_{28}$ is negligible at this temperature. To confirm the stability of $n$ - $\mathrm{C}_{28}$ within the porous silica, a preliminary experiment was performed in which flowing $\mathrm{N}_{2}$ was passed through the pressure vessel at $195^{\circ} \mathrm{C}$ and 14 bar, and the ${ }^{1} \mathrm{H}$ NMR signal of $n-\mathrm{C}_{28}$ was acquired over 3 days of continuous operation. The test result showed no change in signal intensity of $n-\mathrm{C}_{28}$ during this period, thereby confirming no loss of $n-\mathrm{C}_{28}$ due to evaporation and convection. It therefore follows that any change in the $n$ $\mathrm{C}_{28}$ signal in the experiments when water is present is solely due to water phase behaviour within the pores.

The experiment in which condensation into the $n$ - $\mathrm{C}_{28}$-filled pore space is considered first; this experiment is hereafter referred to as the water- $\mathrm{C}_{28}$ experiment. The temperature inside the pressure vessel was first increased to $195{ }^{\circ} \mathrm{C}$ by flowing $\mathrm{N}_{2}$ at $60 \mathrm{NL} \mathrm{h}^{-1}$ through the system in the downflow mode with the system pressure maintained at 2 bar. After reaching the designed temperature, the rig was switched to the upflow mode and liquid water was pumped into the liquid feed line at a liquid flow rate of $50 \mathrm{ml} \mathrm{h}^{-1}$. The liquid water was vaporised to water vapour by the trace heating prior to entering the bottom of the pressure vessel. Water vapour 
exiting from the top of the pressure vessel was cooled down to $90{ }^{\circ} \mathrm{C}$ and condensed to liquid again in the top return line to achieve better pressure control. This operation was maintained for $12 \mathrm{~h}$ to allow temperature and pressure equilibrium inside the pressure vessel. The system was then pressurized with water vapour and the system was allowed to stabilise at pressure steps in the range of 3-13.6 bar corresponding to relative pressures $P / P_{0}$ of $0.2-0.97$; the water saturation vapour pressure $P_{0}$ of 14 bar at $195^{\circ} \mathrm{C}$ was calculated using a literature correlation [34]. A ${ }^{1} \mathrm{H}$ NMR spectrum of the silica bed was acquired every 1 min to check system stabilisation following any pressure step. The system was considered to be stable when there is no significant change in the ${ }^{1} \mathrm{H}$ NMR spectrum; typical stabilisation times were in the range 30-60 min. ${ }^{1} \mathrm{H}$ NMR spectra, PFG NMR measurements of diffusion and ${ }^{1} \mathrm{H} T_{1}$ experiments were then performed, as described in Section 2.3.

Two further experiments were performed. First, the water condensation reference experiment was performed. The experiment setup and procedures were the same as those for the water- $\mathrm{C}_{28}$ experiment. Second, the background signal is acquired under the same experimental conditions. This is acquired by performing the same NMR experiments, and at the same temperature and pressure, as were used for the water condensation reference experiment but with $\mathrm{N}_{2}$ flowing through the pressure vessel. This background NMR signal includes any ${ }^{1} \mathrm{H}$ signal associated with the silica pellets, the glass tube, the pressure vessel and the insulation materials. This background signal was subtracted from the corresponding NMR signal acquired during the water- $\mathrm{C}_{28}$ and water condensation reference experiments.

\subsection{NMR measurements}

The NMR measurements were performed on a Bruker AV 300 spectrometer with a vertical super-wide-bore 7.1 T superconducting magnet, equipped with a 3-axis gradient set providing a maximum gradient strength of $0.77 \mathrm{~T} \mathrm{~m}^{-1}$ in all 3 directions. A birdcage radio frequency (r.f.) coil of diameter $66 \mathrm{~mm}$ was used and was tuned to a resonance frequency of $300.87 \mathrm{MHz}$ for ${ }^{1} \mathrm{H}$ observation. The typical ${ }^{1} \mathrm{H} 90^{\circ}$ pulse duration was $135 \mu \mathrm{s}$.

In the water- $\mathrm{C}_{28}$ experiment, details of the implementation of the NMR experiments are as follows. ${ }^{1} \mathrm{H}$ NMR spectra were acquired using a spin-echo pulse sequence [35] so as to suppress background signal. A recycle time of $30 \mathrm{~s}$ was used with an echo spacing of $400 \mu \mathrm{s} ; 4$ scans were acquired resulting in an acquisition time of $2 \mathrm{~min}$ per spectrum. Three ${ }^{1} \mathrm{H}$ spectra were acquired at each pressure step. Chemical shifts are given relative to the ${ }^{1} \mathrm{H}$ resonance of 
tetramethylsilane. The recycle delay was chosen to ensure that there were no signal saturation effects in the acquired spectra associated with incomplete relaxation between scans for the $n$ $\mathrm{C}_{28}$ species.

$T_{1}$ measurements were measured using a modified saturation recovery sequence that combines the conventional saturation recovery sequence [36] and the spin-echo sequence [35] (Fig. 3 (a)). The spin-echo section was used to supress the background signal as for the spectral data. A 16step relaxation delay list ( $t$ in Fig. 3 (a)) in the range of $1 \mathrm{~ms}-30 \mathrm{~s}$ was used to encode the relaxation times. An echo spacing ( $2 \tau$ in Fig. 3 (a)) of $400 \mu$ s was applied. The $T_{1}$ data were acquired with a recycle time of $4 \mathrm{~s}$ and 4 scans, giving an acquisition time of $12.6 \mathrm{~min}$.

Diffusion coefficients of water and $n-\mathrm{C}_{28}$ were measured with the 13-interval alternative pulsed-gradient stimulated echo (APGSTE) pulse sequence [37] (Fig. 3 (b)) which is designed to eliminate the influence of background magnetic field gradients caused by the magnetic susceptibility differences characteristic of porous media. For measuring water diffusivity, a r.f. pulse spacing $\tau$ of $4.8 \mathrm{~ms}$, a diffusion time $\Delta$ of $20 \mathrm{~ms}$ and a gradient pulse duration $\delta$ of $1 \mathrm{~ms}$ with a gradient ramp time of $0.15 \mathrm{~ms}$ and a gradient stabilisation time of $0.5 \mathrm{~ms}$ were used. A recycle time of $1 \mathrm{~s}$ was used and 8 scans were acquired, yielding an acquisition time of 10.8 $\min$. For measuring the diffusivities of $n-\mathrm{C}_{28}$, two diffusion measurements were performed at each pressure step with a diffusion time $\Delta$ of $50 \mathrm{~ms}$ applied to one measurement and a $\Delta$ of 150 ms applied to the other. This approach was used to confirm no water-oil emulsion formation within the pore space. In both measurements, a r.f. pulse spacing $\tau$ of $4.7 \mathrm{~ms}$, a gradient pulse duration $\delta$ of $6 \mathrm{~ms}$ with a gradient ramp time of $0.15 \mathrm{~ms}$ and a gradient stabilisation time of 0.5 ms was used. The diffusion data of $n-\mathrm{C}_{28}$ were acquired with a recycle time of $2 \mathrm{~s}$ and 4 scans, giving an acquisition time of $5 \mathrm{~min}$ for one $n-\mathrm{C}_{28}$ diffusion dataset. The diffusion data of water and $n$ - $\mathrm{C}_{28}$ were acquired with 64- and 32-step pulsed gradient increments varying in the range of $0.05-70 \mathrm{G} \mathrm{cm}^{-1}$, respectively.

The same methods were used for measurement during the water condensation reference experiment; the only differences being modification of the ${ }^{1} \mathrm{H}$ spectra acquisition parameters since the shorter $T_{1}$ value of water relative to $n$ - $\mathrm{C}_{28}$ enabled a faster recycle time. ${ }^{1} \mathrm{H}$ NMR spectra were acquired with a recycle time of $10 \mathrm{~s}$ and 8 scans which gave an acquisition time of $1.4 \mathrm{~min}$ for a spectrum. $T_{1}$ measurements were made using a 16-step relaxation delay list in the range of $1 \mathrm{~ms}-15 \mathrm{~s}$. A recycle time of $1 \mathrm{~s}$ was used and 8 scans were acquired, giving an 
acquisition time of $11.2 \mathrm{~min}$. Measurement of water diffusivities was made using the same parameters as that for water in the water- $\mathrm{C}_{28}$ experiment.

\section{Data analysis}

The data analysis procedures are described with reference to the water- $\mathrm{C}_{28}$ experiment; the procedure was the same for analysis of the water condensation reference experiment unless stated otherwise. Considering the ${ }^{1} \mathrm{H}$ spectra, prior to any analysis, the background signal was subtracted from the spectra. ${ }^{1} \mathrm{H}$ spectra acquired at various pressures are shown in Fig. 4. A typical ${ }^{1} \mathrm{H}$ NMR spectrum contains 3 peaks with the peaks at $-0.5 \mathrm{ppm}$, at $1.26 \mathrm{ppm}$ and at $3-$ $4 \mathrm{ppm}$ corresponding to water vapour between the silica spheres (inter-pellet water), $n-\mathrm{C}_{28}$, and water within the pores (intra-pellet water), respectively. Identification of the water signal was made by comparing the ${ }^{1} \mathrm{H}$ spectra of the water- $\mathrm{C}_{28}$ system with the ${ }^{1} \mathrm{H}$ spectra acquired in the water condensation reference experiment. The assignment of the water signal to the inter- and intra- pellet environments was possible from the PFG NMR data which were acquired with spectral encoding. The diffusion coefficient values associated with the spectral resonances at $0.5 \mathrm{ppm}$ and 3-4 ppm were $\sim 10^{-6} \mathrm{~m}^{2} \mathrm{~s}^{-1}$ and $\sim 10^{-9} \mathrm{~m}^{2} \mathrm{~s}^{-1}$, respectively. A least squares peak fitting procedure was performed to deconvolve the overlapping peaks; Voigt line-shapes [38] were assumed. The best fits were achieved when the widths of the peaks associated with interpellet water and $n-\mathrm{C}_{28}$ were kept constant when analysing all the spectra. However, a sensitivity analysis to using different fitting methods was performed. These gave similar results and did not influence the conclusions of this study. Following spectral deconvolution, the area under each of the 3 spectral resonances was calculated; these peak areas are proportional to the number of ${ }^{1} \mathrm{H}$ resonances in each chemical environment and therefore are directly proportional to the amount of each species present.

For the $T_{1}$ data acquired in the water- $\mathrm{C}_{28}$ experiment, a background-corrected spectrum $\left(T_{1^{-}}\right.$ weighted spectrum) was obtained for each of the variable delays. The same peak fitting procedure as was used in the spectral analysis was applied to the $T_{1}$-weighted spectra to discriminate the signal of $n-\mathrm{C}_{28}$ and water in the inter- and intra-pellet space. Since only the $T_{1}$ of the $n-\mathrm{C}_{28}$ and intra-pellet water were of interest, and to minimise the error of deconvolution, the total signal of intra-pellet water and $n-\mathrm{C}_{28}$ was obtained by subtracting the signal of inter- 
pellet water from the whole spectrum. The $T_{1}$ values for the intra-pellet water and $n-\mathrm{C}_{28}$ were then obtained by fitting the total signal intensities $S(t)$ of the two species to Eq. (2) which describes the recovery of the magnetisation during a saturation recovery r.f. pulse sequence [36] for a 2-component system:

$$
\frac{S(t)}{S_{T_{0}}}=p_{\mathrm{W}}\left[1-\exp \left(-\frac{t}{T_{1, \mathrm{~W}}}\right)\right]+p_{\mathrm{C}}\left[1-\exp \left(-\frac{t}{T_{1, \mathrm{C}}}\right)\right]
$$

where $S_{T_{0}}$ is the total signal intensity at a full $T_{1}$ recovery, $t$ is the relaxation delay, $p_{\mathrm{W}}$ and $p_{\mathrm{C}}$ $\left(p_{\mathrm{W}}+p_{\mathrm{C}}=1\right)$ are the fractions of the signal of intra-pellet water and $n-\mathrm{C}_{28}$ respectively, and $T_{1, \mathrm{~W}}$ and $T_{1, \mathrm{C}}$ are the relaxation times of intra-pellet water and $n-\mathrm{C}_{28}$ respectively. The $T_{1}$ data obtained in the water condensation experiment were analysed in a similar way with the data fitted to a one-component exponential equation by setting $p_{\mathrm{C}}$ in Eq. (2) to zero.

The PFG NMR data were treated in the same way as the relaxation time data to obtain the individual signal intensities $S(g)$ of the intra-pellet water and $n-\mathrm{C}_{28}$ acquired at different gradient strengths, and the intensities of each species were then fitted to the following equation [39] to obtain the diffusivities:

$$
\frac{S(g)}{S_{D_{0}}}=\exp \left[-\gamma^{2} g^{2} \delta^{2} D\left(\Delta-\frac{\delta}{3}\right)\right]
$$

where $S_{D_{0}}$ is the signal intensity in the absence of gradient, $\gamma$ is the gyromagnetic ratio of ${ }^{1} \mathrm{H}$, $D$ is the diffusivity, $g$ is the strength of the applied magnetic field gradient, $\delta$ is the duration of the applied magnetic field gradient; and $\Delta$ is the diffusion time. The diffusion data acquired in the water condensation experiment in empty silica were analysed in the same way to obtain the diffusivities of inter- and intra-pellet water.

\section{Results}

Figure 5 (a) and (b) show the results of the analysis of the ${ }^{1} \mathrm{H}$ spectra shown in Fig. 4. In Fig. 5 (a) the amount of water present with the pores as a function of water relative pressure for the water- $\mathrm{C}_{28}$ experiment and the water condensation reference experiment are shown. The predicted signal intensity of water dissolved in $n-\mathrm{C}_{28}$ was also calculated using the signal 
intensities of $n-\mathrm{C}_{28}$ and Henry's law, with a Henry's law constant of 40 bar for water in $n-\mathrm{C}_{28}$ at $195{ }^{\circ} \mathrm{C}$ [40]. In Fig. 5 (b) the change in signal intensity and hence amount of $n-\mathrm{C}_{28}$ as a function of water relative pressure is shown. It is seen that at relative pressures of $\sim 0.5 \leq P / P_{0}$ $\leq \sim 0.8$ there is evidence of a small amount of water entering the pore space and displacing $n$ $\mathrm{C}_{28}$ molecules. However, at $P / P_{0}>0.8$ this displacement increases until for $P / P_{0}>0.9$ there is a rapid and significant condensation of water inside the pore space with simultaneous displacement of wax from the pores. Comparing the behaviour of water in the water condensation reference experiment with the water- $\mathrm{C}_{28}$ system behaviour, it is seen that the water condenses into the empty pores more rapidly than when $n-\mathrm{C}_{28}$ is present but the trend as a function of water relative pressure is the same. The data for the water condensation reference experiment are consistent with multiple adsorbed layers of water forming within the pore space followed by further rapid condensation to complete pore-filling [41]. The prediction of the Henry's Law calculation clearly shows that the ${ }^{1} \mathrm{H}$ NMR signal intensity is inconsistent with water simply dissolved in the wax present inside the pore space.

Figure 6 (a) and (b) show the $T_{1}$ relaxation times of water and $n-\mathrm{C}_{28}$ acquired as a function of the relative pressure of water, respectively. It is clearly seen that the relaxation times, which reflect the physical environment of the species, follow the same trend as was observed in Fig. 5; there is very little change in $T_{1}$ until $P / P_{0} \sim 0.8$ and a more rapid change in $T_{1}$ for $P / P_{0}>$ 0.9 .

Figure 7 (a) and (b) present the diffusivities of water and $n-\mathrm{C}_{28}$ obtained in the water- $\mathrm{C}_{28}$ experiment, respectively. In Fig. 7 (a), only the water diffusivities measured at high pressure $P / P_{0}>0.90$ are presented and the results obtained at lower pressure are not shown due to the larger error caused by the low signal-to-noise ratio associated with the very small ${ }^{1} \mathrm{H}$ signal intensities acquired at these pressures. It is observed in Fig. 7 (a) that at $P / P_{0}>0.90$ the diffusivity of water is not sensitive to pressure and is stable at $5.9 \times 10^{-9} \mathrm{~m}^{2} \mathrm{~s}^{-1}$ which is smaller than both the self-diffusivity of water at $195{ }^{\circ} \mathrm{C}$ measured by NMR $\left((18 \pm 0.20) \times 10^{-9} \mathrm{~m}^{2} \mathrm{~s}^{-1}\right)$ and that calculated by the Wilke-Chang correlation [42] and then scaled with a tortuosity factor of 1.6 determined using pulsed-field gradient NMR. The measurement of tortuosity factor used followed the method of Hollewand and Gladden [43]. Figure 7 (b) shows the diffusivities of $n$ $\mathrm{C}_{28}$ obtained with diffusion times $\Delta$ of $50 \mathrm{~ms}$ and $150 \mathrm{~ms}$, and it is seen that the data obtained with both diffusion times are consistent, showing no evidence of emulsion formation [44]. It is also seen in Fig. 7 (b) that the diffusivity of $n-\mathrm{C}_{28}$ varies in a similar way to that of the ${ }^{1} \mathrm{H}$ 
spectral intensities and $T_{1}$ of $n$ - $\mathrm{C}_{28}$ with the diffusivity increasing slowly at $P / P_{0}<0.80$ from $0.80 \times 10^{-9}$ to $0.92 \times 10^{-9} \mathrm{~m}^{2} \mathrm{~s}^{-1}$ and rapidly rising to around $1.1 \times 10^{-9} \mathrm{~m}^{2} \mathrm{~s}^{-1}$ at $P / P_{0}>0.90$.

\section{Discussion}

\subsection{Capillary condensation of water in $C_{28}$-saturated pores}

Figure 5 (a) showed that the signal intensity of water in $\mathrm{C}_{28}$-saturated silica shows very similar behaviour to that of water condensation in the reference experiment. Further, comparison of these data with Fig. 1 shows that this behaviour as a function of pressure is very similar to that of the $\mathrm{N}_{2}$ adsorption isotherm, where again a sudden increase in the amount of condensed nitrogen occurs in a relative pressure range of $0.8<P / P_{0}<1$. These data therefore provide evidence to suggest that capillary condensation of water in $\mathrm{C}_{28}$-saturated silica is occurring. The capillary condensation in systems of immiscible liquids was reported by Christenson [45] who observed water condensation in a confined space between two mica surfaces filled by OMCTS oil. A thermodynamic description of this system was developed later by Evans and Marconi [46] by assuming that the surface wetting phase is pure water and the oil-rich phase is a dilute solution of water in oil. The capillary condensation of water in oil can then be described by the following equation:

$$
\ln \left(\frac{f_{\mathrm{W}}}{f_{\mathrm{W}, \mathrm{b}}}\right)=-\frac{2 V_{\mathrm{m}, \mathrm{W}} \gamma}{R T r}
$$

where $f_{\mathrm{W}}$ and $f_{\mathrm{W}, \mathrm{b}}$ are the fugacities of water in the oil-rich phase in contact with water with a curved and flat interface, respectively, $V_{\mathrm{m}, \mathrm{W}}$ is the molar volume of water and $\gamma$ is the interfacial tension between water and oil, $R$ is the ideal gas constant, $T$ is temperature and $r$ is the pore radius. Given that the form of Eq. (4) is analogous to that of the conventional Kelvin equation, this analysis suggests that it is appropriate to interpret the data shown in Fig. 5 (a) as being consistent with a capillary condensation process occurring as water enters the waxsaturated pores.

From the perspective of capillary condensation, the variation of signal intensity of water in $\mathrm{C}_{28}$ saturated silica (Fig. 5 (a)) is easily understood. The initial slow increase at low pressure is explained by the increase in the thickness of the surface adsorbed layer of water, with the rapid increase in water signal intensity occurring at the onset of capillary condensation. Insights into 
the behaviour of water molecules during this process are revealed by $T_{1}$ relaxation time (Fig. 6 (a)) and diffusivity (Fig. 7 (a)) data recorded for water. $T_{1}$ relaxation time is used as an indicator of surface interaction strength with a small $T_{1}$ indicating large interaction strength and a large $T_{1}$ indicating a weak interaction [29,30]. For the $T_{1}$ of water in $\mathrm{C}_{28}$-saturated silica shown in Fig. 6 (a), the relatively small $T_{1}$ values of $0.1-0.3 \mathrm{~s}$ at $P / P_{0}<0.90$ imply that water molecules are experiencing a relatively strong interaction with the surface. Thus these data are consistent with phase separation occurring within the pore even at low water concentrations, with water forming a surface layer. It is also seen at $P / P_{0}<0.90$ that the $T_{1}$ values of water in $\mathrm{C}_{28}$-saturated silica are similar to, but slightly larger than, those obtained in the reference experiment when water is adsorbed into empty pores; this is consistent with the water-surface interaction being reduced in the water- $\mathrm{C}_{28}$ experiment by the presence of $n-\mathrm{C}_{28}$ molecules which might be present in small amount at the pore surface. For $P / P_{0}>0.90$ during capillary condensation the $T_{1}$ in both the water- $\mathrm{C}_{28}$ and water condensation reference experiment increases as expected from the two fraction fast exchange model [30] in which molecules in the bulk pore space (associated with a longer $T_{1}$ ) exchange, over the timescale of the NMR relaxation time measurement, with molecules in the surface-influenced layer which are associated with a shorter $T_{1}$ value. Albeit on the basis of just two data points, at $P / P_{0}>0.90$ when water condensation occurs, the $T_{1}$ of water in initially $\mathrm{C}_{28}$-saturated pores does become smaller than that of water in empty silica indicating stronger surface interaction of condensed water with the pore surface when water co-exists with $n-\mathrm{C}_{28}$ within the pore space. Thus the $T_{1}$ data are also consistent with motion of water molecules being confined between the pore surface and an oil-rich phase that occupies the central pore space. The $T_{1}$ data are consistent with the diffusion data shown in Fig. 7. Figure 7 (a) shows that the diffusivity of water in $\mathrm{C}_{28}$-saturated pores when capillary condensation has occurred is consistently smaller than the water self-diffusivity in the water condensation reference experiment. There exists both measurement and simulation evidence in support of adsorbate diffusivity varying with the distance from the pore surface, with slower diffusivity closer to the pore surface [47-49]; however the data shown in Fig. 7 (a) confirm that the presence of $n-\mathrm{C}_{28}$ within the pore decreases the water diffusivity even more than is found when pure water condenses within an empty pore. This will arise both from the presence of remaining $n-\mathrm{C}_{28}$ slowing down the motion of water molecules within the water-rich surface layer and the confinement effect of the water-rich surface layer between the pore surface and the oil-rich phase in the centre of the pore. These results are in excellent agreement with the observation from the simulation of water- $\mathrm{C}_{28}$ equilibrium in a $5.5 \mathrm{~nm}$ width $\mathrm{TiO}_{2}$ pore at $200{ }^{\circ} \mathrm{C}$ reported 
by Papavasileiou et al. [25] who showed that even under conditions close to full water saturation with water mole fraction of 0.9655 in the pore, the water molecules remained in a phase separated layer adjacent to the pore surface, with $n-\mathrm{C}_{28}$ molecules present in the pore centre. The restriction of the motion of water molecules in the surface layer adjacent to the pore surface was also observed in that work. The diffusivity of $n-\mathrm{C}_{28}$ shown in Fig. 7 (b) shows a similar behaviour to the $T_{1}$ data in Fig. 6 (b); both reflect the mobility of $n$ - $\mathrm{C}_{28}$ molecules. The rapid increase in the $D$ and $T_{1}$ of $n-\mathrm{C}_{28}$ at $P / P_{0}>0.9$ is consistent with $n-\mathrm{C}_{28}$ molecules being displaced from the pore surface and therefore experiencing a weaker interaction with the surface.

\subsection{Implications of observed wax-water behaviour on Fischer-Tropsch synthesis}

Whilst the data reported here are not recorded during Fischer-Tropsch synthesis it is interesting to consider the implications of the wax-water phase behaviour observed. In this section, estimates of the percentage of the volume (hereafter referred to as \% volume) of the pore space occupied by water, and the percentage of the pore surface (hereafter referred to as $\%$ surface) covered by water are calculated and the results used to consider implications for the behaviour of Fischer-Tropsch synthesis catalysts.

Assuming a model for capillary condensation within the pore space in which water will condense in the smallest pores first, Figure 8 shows both the $\%$ volume and $\%$ surface of the pore space occupied by water as a function of water relative pressure based on the experimental data presented in Fig. 5 and the nitrogen adsorption data presented in Fig. 1. The data shown in Fig. 8 are calculated using the fact that the acquired signal intensities of each chemical species are directly proportional to the number of nuclei (i.e., atoms) of these species in the system. Therefore, the ratio of the volumes of water $\left(V_{\mathrm{W}}\right)$ and $n-\mathrm{C}_{28}\left(V_{\mathrm{C}}\right)$ within the pore space in terms of the molar mass $M$, molar density $\rho$, and signal intensity $S$, (taken from Fig. 5) of the two chemical species is given by:

$$
\frac{V_{\mathrm{W}}}{V_{\mathrm{C}}}=\left[\left(\frac{M_{\mathrm{W}}}{2 \rho_{\mathrm{W}}}\right) \cdot\left(\frac{58 \rho_{\mathrm{C}}}{M_{\mathrm{C}}}\right)\right] \cdot \frac{S_{\mathrm{W}}}{S_{\mathrm{C}}}
$$

where the subscripts $\mathrm{w}$ and $\mathrm{c}$ indicate water and $n-\mathrm{C}_{28}$ respectively. The numbers 2 and 58 are the numbers of hydrogen atoms in a water and a $n-\mathrm{C}_{28}$ molecule respectively. Hence, the \% volume of water as a function of the relative pressure of water is obtained; values for the density 
of water $\left(0.87 \mathrm{~g} \mathrm{ml}^{-1}\right)$ and $n-\mathrm{C}_{28}\left(0.69 \mathrm{~g} \mathrm{ml}^{-1}\right)$ at $195{ }^{\circ} \mathrm{C}$ were taken from Perry et al. [34] and Rodden et al. [50], respectively. The \% surface occupied by water is then obtained by calculating the pore-size distribution (PSD) of the porous silica used by applying the BarrettJoyner-Halenda (BJH) method [41] to the nitrogen desorption data (Fig. 1) and applying the appropriate form of the Kelvin equation [52] assuming a cylindrical pore shape. The BJH method assumes that capillary condensation occurs in the smallest pores first and the $\%$ surface occupied by liquid for all values of $\%$ volume filled with liquid is calculated from the cumulative PSD and is shown in the inset of Fig. 8. Finally, we assume that the water condensation will also occur in the smallest pores first as $P / P_{0}$ is increased and so the relationship between the $\%$ volume of water and the $\%$ surface occupied by water can be calculated from the inset graph in Fig. 8. For each step in partial pressure, $P / P_{0}$, in Fig. 5 the total volume of water condensed is calculated from Eq. (5) and hence the \% surface occupied by water is calculated and is plotted in Fig. 8. It is noted that in deriving the data shown in Fig. 8 the relationship between the $\%$ volume and \% surface occupancies of water obtained using the BJH method [41] assumes the whole pore volume is occupied by condensate. The estimate of the fraction of the surface occupied by water is therefore an under-estimate since water layers will exist within oil-rich pores before the onset of water condensation.

Inspection of Fig. 8 shows that the condensed water occupies significant amount of pore volume and pore surface area at $P / P_{0} \geq 0.85$, reaching volume and surface occupancies of $\sim 5 \%$ and $10 \%$, respectively, at $P / P_{0}=0.85$. The rapid and significant condensation for $P / P_{0}>0.9$ is associated with \% volume and \% surface occupancies reaching in excess of $30 \%$ and $40 \%$ respectively at $P / P_{0}=0.97$. This analysis suggests the following: (i) water condensation in the wax-filled pores will be sensitive to pore size; and (ii) for a given pore size, the sensitivity of the catalyst activity to the amount of water present may vary significantly as the catalyst pore space moves from a state of the pore surface becoming increasingly covered with water before full condensation of the water within the pores.

The confirmation that water can condense within wax-saturated pores, thereby displacing the wax from the pore surface provides potential insights and understanding of previously reported catalyst behaviour. The following is not a detailed summary of work reported in LTFT but attempts to draw out some of the common themes of those works. Most importantly, in discussing the insights that might be obtained from the results presented herein, it is relevant to note that typical operating conditions in these works lie in the temperature range of 200- 
$220{ }^{\circ} \mathrm{C}$ which gives a water saturation vapour pressure $P_{0}$ of $15-23$ bar $[9,13,14,16-19]$. The water partial pressure in the reactors was typically reported to be less than 10 bar, resulting in a relative pressure $P / P_{0}$ of $0.3-0.5$ in the reactors. This value is obviously significantly less than the relative pressures over which significant water condensation in the pores is occurring in the present study. However, the observation has already been made that the amount of water contacting the pore surface is expected to be greater at lower relative pressures than shown in Fig. 8; this would suggest that the presence of water within the catalyst could influence catalytic behaviour at partial pressures less than 0.5 in Fig. 8. Perhaps, more significantly, as noted by Iglesia [12], mass transfer limitations under Fischer-Tropsch synthesis reaction conditions, will lead to a concentration gradient in the mole fraction of water present between the centre of the catalyst pellet and the inter-pellet gas phase. As discussed in that work, these diffusional restrictions in the removal of water formed lead to significantly higher water fugacities near the centre of the catalyst pellets than in the inter-pellet gas phase. Given the proportionality, via Henry's constant, of pressure (or fugacity) to the relevant water mole fraction term (i.e. within the pores or in the inter-pellet space), a concentration ratio of water in the pores to that in the inter-pellet space of only 2-3 would imply that for reactor conditions (i.e., inter-pellet space conditions) of $P / P_{0}$ of $0.3-0.5$, the water within the pores will exist in a fugacity regime $f_{\mathrm{W}} / f_{\mathrm{W}, \mathrm{b}}>0.9$. This would imply that the catalysts reported in these earlier works could experience the condensation behaviour reported in this work.

Many works have considered the effects of pore size and co-fed water on catalyst performance. The pore size of the catalyst has been reported to have a significant effect on the influence of co-fed water on FT catalyst performance [16,18,19]. For example, a more pronounced deactivation was observed for Co supported on a $\gamma-\mathrm{Al}_{2} \mathrm{O}_{3}$ of pore diameter $11.6 \mathrm{~nm}$ compared to Co supported on a $\gamma-\mathrm{Al}_{2} \mathrm{O}_{3}$ of pore diameter $18.3 \mathrm{~nm}$ [16]. Similarly, catalyst deactivation by water has been reported to be more significant on Co catalysts supported on $\gamma-\mathrm{Al}_{2} \mathrm{O}_{3}$ of small pore size $(\leq 10 \mathrm{~nm})$ than on Co catalysts supported by $\alpha-\mathrm{Al}_{2} \mathrm{O}_{3}, \mathrm{SiO}_{2}$ and $\mathrm{TiO}_{2}$ of much larger pore size $[18,19]$. The effect of support on the water effect has often been explained by the cobalt cluster size which is affected by the pore size and metal-support interaction of different supports [19,53,54]. However, Iglesia [12] suggested that the presence of a condensed water phase might be more consistent with such observations. Amongst many observations reported in [12], data for Co catalysts supported on small and large pore $\mathrm{SiO}_{2}$ were presented. In particular, it was observed that at a water partial pressure $<3$ bar the turnover rates of the large- 
pore catalyst increased with increasing amount of water at first, with the increase in activity becoming weaker at higher water partial pressure; these observations were assigned to water concentration effects becoming 'saturated' at high water concentration. It was also observed that the water effect on the small-pore catalyst reached the saturation state much more easily compared to the large-pore catalyst due to the condensation of water in the small-pore catalyst at lower water partial pressures. Such observations are readily explained by the data reported in Figs. 5 and 8; the onset of the 'deactivated' and 'saturated' states being consistent with the presence of significant capillary condensation within the catalyst pores.

Capillary condensation within the pores also offers an explanation for results reported by others focusing on the effects of co-fed water. For example, as stated earlier, it has been reported that the deactivation of Co catalysts strongly depends on the amount of water present in the system with reversible water effects observed at the amount of co-fed water $<25 \mathrm{vol} \%$ in the feed gas mixture and a sudden change to permanent deactivation observed at the co-fed water amount $\geq 25 \%[9,14,16]$. It has also been observed from the results on a $\mathrm{Co} / \mathrm{Al}_{2} \mathrm{O}_{3}$ catalyst by Fratalocchi et al. [17] that addition of water to the feed causes deactivation of the catalyst with a deactivation rate of $0.001 \mathrm{~h}^{-1}$ when the concentration of co-fed is $4.9 \mathrm{vol} \%$ and $15.2 \mathrm{vol} \%$, with the deactivation rate increasing to $0.004 \mathrm{~h}^{-1}$ when the co-fed water concentration increases to $28 \mathrm{vol} \%$. The identification of reversible water effects is particularly interesting since it would be consistent with a capillary condensation cycle. However, these earlier observations also suggest that at high water partial pressures reversibility is lost. The possible formation of hydrocarbon-water emulsions stabilised by oxygenates has been discussed by earlier workers [12] and perhaps the formation of such emulsions marks the point at which the effects of capillary condensation are no longer reversible. This is the subject of ongoing work.

Of course, in making the above comments it is not suggested that previously reported correlations between changes in catalyst characteristics and hence activity and selectivity as a function of pore size and the amount of water present are incorrect; the presence of water may indeed influence metal Co crystallite characteristics. For example, it is noted that the deactivation of Co catalysts by water is commonly related to the change of the morphology of surface Co. At low amounts of co-fed water, the adsorbed water may affect the catalyst performance by causing reversible oxidation of cobalt particles [55] or via a reversible kinetic process [4]. Irreversible deactivation of the catalyst by mechanisms such as the sintering of cobalt particles [7,55] or the formation of a mixed metal oxide phase [4,55] have also 
previously been reported to be assisted and accelerated by water; this irreversible condensation may be associated with the onset of condensation of water within the pore space.

\section{Conclusion}

This work presents an operando study of the phase behaviour of water in a porous silica saturated by $n-\mathrm{C}_{28}$ under the conditions relevant to FT synthesis using multiple NMR techniques. Capillary condensation of water was observed in the $\mathrm{C}_{28}$-saturated silica at a relative pressure range almost identical to that for the water condensation into the empty silica of an as-received silica. The surface interaction and diffusion of water and $n-\mathrm{C}_{28}$ molecules during the process of water condensation were studied using magnetic resonance nuclear spinlattice relaxation time and molecular diffusion measurement techniques. During the condensation process, the water was found to displace the $n-\mathrm{C}_{28}$ from the pore surface and to exist as a highly water-rich layer confined between the pore surface and an oil-rich phase which occupies the central pore space. This confined water layer was characterised by a strong surface interaction and a slower diffusivity than was observed for single-component water within the same pore space. The NMR data for the $n-\mathrm{C}_{28}$ species confirmed that $n-\mathrm{C}_{28}$ was displaced by the water condensate in pores and that the hydrocarbon became isolated from the pore surface by water, thereby causing a reduction of the surface interaction and an increase in diffusivity of $n-\mathrm{C}_{28}$ species as condensation proceeded.

The pore volume and surface occupied by water during the process of condensation were calculated and it was observed that the water condensation can result in up to $\sim 40 \%$ of the pore surface being covered with water for the porous silica studied. A brief survey of previously published studies of LTFT with particular focus on the effect of pore size and co-fed water on catalyst performance suggest that capillary condensation of water within these catalysts is consistent with the observations in those works. 


\section{Acknowledgements}

The authors would like to thank Shell Global Solutions International B.V. for funding this work. JLB also thanks both the Cambridge Commonwealth Trust and the Institution of Chemical Engineers (Andrew Fellowship) for funding. We also wish to thank H. Oosterbeek and H.T.M. Smits for useful discussions and help in the designing and commissioning of the operando reactor. 


\section{References}

[1] D.A. Wood, C. Nwaoha, B.F. Towler, J. Nat. Gas Sci. Eng. 9 (2012) 196-208.

[2] S.S. Ail, S. Dasappa, Renew. Sustain. Energy Rev. 58 (2016) 267-286.

[3] M.E. Dry, Catal. Today 71 (2002) 227-241.

[4] G. Jacobs, T.K. Das, P.M. Patterson, J. Li, L. Sanchez, B.H. Davis, Appl. Catal. A Gen. 247 (2003) 335-343.

[5] G. Jacobs, P.M. Patterson, T.K. Das, M. Luo, B.H. Davis, Appl. Catal. A Gen. 270 (2004) 65-76.

[6] P.J. van Berge, J. van de Loosdrecht, S. Barradas, A.M. van der Kraan, Catal. Today 58 (2000) 321-334.

[7] G.L. Bezemer, T.J. Remans, A.P. van Bavel, A.I. Dugulan, J. Am. Chem. Soc. 132 (2010) 8540-8541.

[8] M. Sadeqzadeh, S. Chambrey, S. Piche, P. Fongarland, F. Luck, D. Curulla-Ferre, D. Schweich, J. Bousquet, A.Y. Khodakov, Catal. Today 215 (2013) 52-59.

[9] J. Li, X. Zhan, Y. Zhang, G. Jacobs, T. Das, B.H. Davis, Appl. Catal. A Gen. 228 (2002) 203-212.

[10] C.J. Bertole, C.A. Mims, G. Kiss, J. Catal. 210 (2002) 84-96.

[11] D.D. Hibbitts, B.T. Loveless, M. Neurock, E. Iglesia, Angew. Chemie - Int. Ed. 52 (2013) 12273-12278.

[12] E. Iglesia, Appl. Catal. A Gen. 161 (1997) 59-78.

[13] S. Krishnamoorthy, M. Tu, M.P. Ojeda, D. Pinna, E. Iglesia, J. Catal. 211 (2002) 422433.

[14] J. Li, G. Jacobs, T. Das, Y. Zhang, B. Davis, Appl. Catal. A Gen. 236 (2002) 67-76.

[15] M. Claeys, E. van Steen, Catal. Today 71 (2002) 419-427.

[16] O. Borg, S. Storsater, S. Eri, H. Wigum, E. Rytter, A. Holmen, Catal. Letters 107 (2006) 95-102.

[17] L. Fratalocchi, C.G. Visconti, L. Lietti, G. Groppi, E. Tronconi, E. Roccaro, R. Zennaro, Catal. Sci. Technol. 6 (2016) 6431-6440.

[18] S. Logdberg, M. Boutonnet, J.C. Walmsley, S. Jaras, A. Holmen, E.A. Blekkan, Appl. Catal. A Gen. 393 (2011) 109-121.

[19] S. Storsæter, O. Borg, E.A. Blekkan, A. Holmen, J. Catal. 231 (2005) 405-419.

[20] W. Ma, G. Jacobs, D.E. Sparks, R.L. Spicer, B.H. Davis, J.L.S. Klettlinger, C.H. Yen, Catal. Today 228 (2014) 158-166.

[21] B. Coasne, A. Galarneau, R.J.M. Pellenq, F. Di Renzo, Chem. Soc. Rev. 42 (2013) 4141-4171.

[22] S.K. Singh, A. Sinha, G. Deo, J.K. Singh, J. Phys. Chem. C 113 (2009) 7170-7180.

[23] V.S. Ermolaev, K.O. Gryaznov, E.B. Mitberg, V.Z. Mordkovich, V.F. Tretyakov, Chem. Eng. Sci. 138 (2015) 1-8.

[24] V.S. Ermolaev, V.Z. Mordkovich, I.G. Solomonik, Theor. Found. Chem. Eng. 44 (2010) 660-664.

[25] K.D. Papavasileiou, Z.A. Makrodimitri, L.D. Peristeras, J. Chen, G.P. van der Laan, I. Rudra, A. Kalantar, I.G. Economou, J. Phys. Chem. C 120 (2016) 24743-24753.

[26] C. Erkey, J.B. Rodden, A. Akgerman, Energy \& Fuels 4 (1990) 275-276.

[27] J. Kärger, R. Valiullin, Chem. Soc. Rev. 42 (2013) 4172-4197.

[28] D. Weber, A.J. Sederman, M.D. Mantle, J. Mitchell, L.F. Gladden, Phys. Chem. Chem. Phys. 12 (2010) 2619-2624.

[29] C. D’Agostino, J. Mitchell, L.F. Gladden, M.D. Mantle, J. Phys. Chem. C 116 (2012) 8975-8982. 
[30] G. Liu, Y. Li, J. Jonas, J. Chem. Phys. 95 (1991) 6892-6901.

[31] S.T. Roberts, M.P. Renshaw, M. Lutecki, J. McGregor, A.J. Sederman, M.D. Mantle, L.F. Gladden, Chem. Commun. 49 (2013) 10519-10521.

[32] L. Baker, M.P. Renshaw, M.D. Mantle, A.J. Sederman, A.J. Wain, L.F. Gladden, Appl. Catal. A Gen. 557 (2018) 125-134.

[33] J.S. Chickos, W. Hanshaw, J. Chem. Eng. Data 49 (2004) 77-85.

[34] R.H. Perry, D.W. Green, J.O. Maloney, Perry's Chemical Engineers' Handbook, 7th ed., McGrawHill Publishing, New York, 1997.

[35] E. Hahn, Phys. Rev. 80 (1950) 580-594.

[36] J.L. Markley, W.J. Horsley, M.P. Klein, J. Chem. Phys. 55 (1971) 3604-3605.

[37] R.M. Cotts, M.J.R. Hoch, T. Sun, J.T. Markert, J. Magn. Reson. 83 (1989) 252-266.

[38] E.E. Whiting, J. Quant. Spectrosc. Radiat. Transf. 8 (1968) 1379-1384.

[39] E.O. Stejskal, J.E. Tanner, J. Chem. Phys. 42 (1965) 288-292.

[40] B.B. Breman, A.A.C.M. Beenackers, E.W.J. Rietjens, R.J.H. Stege, J. Chem. Eng. Data 39 (1994) 647-666.

[41] E.P. Barrett, L.G. Joyner, P.P. Halenda, J. Am. Chem. Soc. 73 (1951) 373-380.

[42] C.R. Wilke, P. Chang, AIChE J. 1 (1955) 264-270.

[43] M.P. Hollewand, L.F. Gladden, Chem. Eng. Sci. 50 (1995) 309-326.

[44] K.G. Hollingsworth, M.L. Johns, J. Colloid Interface Sci. 258 (2003) 383-389.

[45] H.K. Christenson, J. Colloid Interface Sci. 104 (1985) 234-249.

[46] R. Evans, U.M.B. Marconi, J. Chem. Phys. 86 (1987) 7138-7148.

[47] I.C. Bourg, C.I. Steefel, J. Phys. Chem. C 116 (2012) 11556-11564.

[48] A. Pajzderska, M.A. Gonzalez, J. Mielcarek, J. Wasicki, J. Phys. Chem. C 118 (2014) 23701-23710.

[49] T.G.A. Youngs, D. Weber, L.F. Gladden, C. Hardacre, J. Phys. Chem. C 113 (2009) 21342-21352.

[50] J.B. Rodden, C. Erkey, A. Akgerman, J. Chem. Eng. Data 33 (1988) 450-453.

[51] R.L. Fogelson, E.R. Likhachev, Tech. Phys. 46 (2001) 1056-1059.

[52] H.-J. Butt, K. Graf, M. Kappl, Physics and Chemistry of Interfaces, Wiley-VCH GmbH \& Co. KGaA, Weinheim, 2003.

[53] G. Jacobs, T.K. Das, Y. Zhang, J. Li, G. Racoillet, B.H. Davis, Appl. Catal. A Gen. 233 (2002) 263-281.

[54] A.Y. Khodakov, Catal. Today 144 (2009) 251-257.

[55] C.E. Kliewer, S.L. Soled, G. Kiss, Catal. Today (2018)

http://doi.org/10.1016/j.cattod.2018.05.021 


\section{Figure captions}

Figure 1. Nitrogen adsorption/desorption isotherms recorded for the porous silica used.

Figure 2. Schematic of the pilot-scale flow loop. 1. Feed vessel. 2. HPLC piston pump. 3. Ceramic column. 4. NMR magnet. 5. Glass tube. 6. Waste vessel. For clarity, the parts of the rig equipped with trace heating are highlighted in red (online version only).

Figure 3. NMR pulse sequences used: (a) The modified saturation recovery sequence for $T_{1}$ measurement. (b) The APGSTE sequence for diffusion measurement. The pulse sequence parameters are identified in the text.

Figure 4. NMR spectra of the system acquired as a function of water relative pressure. The peaks located at $-0.5 \mathrm{ppm}, 1.26 \mathrm{ppm}$ and $3-4 \mathrm{ppm}$ are associated with inter-pellet water, $n-\mathrm{C}_{28}$ and intrapellet water respectively. Magnetic resonance imaging confirmed that displaced wax drains to the bottom of the bed and is not held-up in the inter-pellet space. Chemical shifts are referenced to the ${ }^{1} \mathrm{H}$ resonance of tetramethylsilane. The resonances associated with intra-pellet water and $n-\mathrm{C}_{28}$ increase and decrease respectively as water relative pressure increases.

Figure 5. Changes in the integrated ${ }^{1} \mathrm{H}$ magnetic resonance signal intensity of intra-pellet water and $n$ $\mathrm{C}_{28}$ wax as a function of water relative pressure, obtained from Fig. 4. (a) Data are shown for water, in the water- $\mathrm{C}_{28}$ experiment (-- --); the water condensation reference experiment (--०--); and the

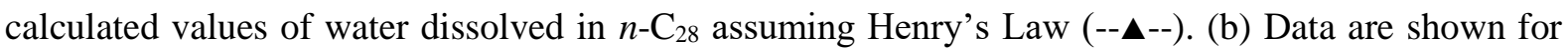
wax for the water- $\mathrm{C}_{28}$ experiment (-- --). The dashed lines are shown to guide the eye. The error bar represents the standard error of each data point, and all data points are subject to a horizontal error bar of \pm 0.02 which is not shown.

Figure 6. $T_{1}$ values of water and $n-\mathrm{C}_{28}$ wax as a function of water relative pressure. (a) Data are shown

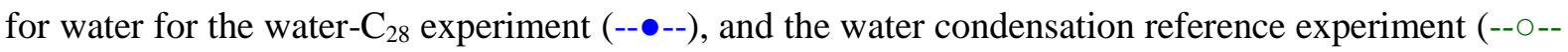
). (b) Data are shown for wax for the water-C $\mathrm{C}_{28}$ experiment (-- $\left.\bullet-\right)$. The dashed lines are shown to guide the eye. The error bar represents the standard error of each data point, and all data points are subject to a horizontal error bar of \pm 0.02 which is not shown. 
Figure 7. Diffusivity of water and $n-\mathrm{C}_{28}$ as a function of water relative pressure. (a) Data are shown for water for the water- $\mathrm{C}_{28}$ experiment $(\bullet)$. The solid horizontal line indicates the water self-diffusivity measured by PFG NMR at $P / P_{0}=1$ in the water condensation reference experiment. This measured diffusivity is subject to a standard error of $\pm 0.20 \times 10^{-9} \mathrm{~m}^{2} \mathrm{~s}^{-1}$. The dashed horizontal line indicates the self-diffusivity of water calculated using the Wilke-Chang correlation [42] and scaled by the tortuosity factor of 1.6 measured by PFG NMR using water as the probe molecule at $20{ }^{\circ} \mathrm{C}$. A density of 0.048 mol ml-1 [34] and a viscosity of $0.14 \mathrm{cp} \mathrm{[51]} \mathrm{of} \mathrm{liquid} \mathrm{water} \mathrm{at} 195{ }^{\circ} \mathrm{C}$ were used in the calculation. (b) Data are shown for $n-\mathrm{C}_{28}$ with the data acquired with $\Delta=50 \mathrm{~ms}(--\bullet--)$, and $\Delta=150 \mathrm{~ms}(--\bullet--)$. The dashed lines are shown to guide the eye. The error bar represents the standard error of each data point, and all data points are subject to a horizontal error bar of \pm 0.02 which is not shown.

Figure 8. Estimated percentages of the pore volume and surface occupied by water condensate. The inset shows the relationship between the percentage of the pore surface and volume occupied by condensate for the silica used in this work, based on the BJH analysis of the $\mathrm{N}_{2}$ desorption data shown in Fig. 1. 